Research Paper

\title{
Ethyl acetate fraction of Huogu formula inhibits adipogenic differentiation of bone marrow stromal cells via the BMP and Wnt signaling pathways
}

\author{
Xiangying Kong ${ }^{*}$, Xiaomin Li1 ${ }^{*}$, Cun Zhang ${ }^{1}$, Liuluan Zhu ${ }^{2}$, Chunfang Liu${ }^{1}$, Qingxia Qin ${ }^{1}$, Cuiling Liu ${ }^{1}$, \\ Qianqian Wang ${ }^{1}$, Jia Zhu ${ }^{3}$, Xuan $W_{u^{1}}$, Hongye Wan ${ }^{1}$, Weiheng Chen ${ }^{3 凶}, \mathrm{Na} \mathrm{Lin}^{1 凶}$ \\ 1. Institute of Chinese Materia Medica, China Academy of Chinese Medical Sciences, 100700 Beijing, China. \\ 2. Institute of Infectious Diseases, Beijing Ditan Hospital, Capital Medical University, Beijing, China; Beijing Key Laboratory of Emerging Infectious Diseases, \\ 100015 Beijing, China. \\ 3. Wangiing Hospital, China Academy of Chinese Medical Sciences, 100102 Beijing, China \\ * These authors as first authors contributed equally to this article. \\ $\bowtie$ Corresponding authors: Prof. Na Lin nlin@icmm.ac.cn Tel: +861064014411-2869; Fax: +861064013996. Prof. Weiheng Chen, drchenweiheng@163.com
}

(c) Ivyspring International Publisher. This is an open access article distributed under the terms of the Creative Commons Attribution (CC BY-NC) license (https://creativecommons.org/licenses/by-nc/4.0/). See http://ivyspring.com/terms for full terms and conditions.

Received: 2016.11.21; Accepted: 2017.01.23; Published: 2017.04.07

\begin{abstract}
Elevated adipogenesis of bone marrow stromal cells (BMSCs) is closely associated with non-traumatic osteonecrosis of femoral head (ONFH). Our previous studies have shown that Huogu (HG) formula was effective both in clinic experience and experimental ONFH. How HG impacts the differentiation of BMSCs and what is the underlying molecular mechanism remain largely unknown. Our results showed that ethyl acetate extract of HG (HGE) significantly decreased the adipocyte differentiation as determined by oil red staining, while slightly increased the ALP activity. Investigation of the molecular mechanism revealed that HGE could inhibit the mRNA and protein expression of peroxisome proliferators-activated receptor (PPAR) $\gamma$, lipoprotein lipase (LPL) and adipocyteprotein2 (AP2). Interestingly, the inhibition of adipogenic differentiation in BMSCs by HGE could be restored by DKK-1, an inhibitor of Wnts. However, Noggin (an inhibitor of BMPs) displayed an additive role with HGE in suppressing the expression of PPARY, LPL, and AP2. Furthermore, the bone marrow fat formation, as well as the expression of Wnt3a and PPAR $\gamma$, was effectively regulated by HGE in the steroid-induced ONFH rats. Our results demonstrated that $\mathrm{HGE}$ treatment significantly inhibited adipogenesis and slightly promoted osteogenesis of BMSCs through regulating the BMP and Wnt pathways. The findings shed lights on the molecular mechanism of HGE in the inhibition of adipogenesis and provide scientific rationale for its clinical application of HGE in the treatment of ONFH.
\end{abstract}

Key words: Adipogenic differentiation; Osteonecrosis; Bone marrow stromal cells; Huogu Formula; Chinese medicine

\section{Introduction}

Glucocorticoids have been widely applied in clinical treatment of inflammation and autoimmune diseases. However, steroid associated osteonecrosis frequently occurs as a result of high-dose corticosteroid treatments [1, 2]. Previous studies indicated that steroid caused significant enlargement of the adipocyte size and fat accumulation in the bone marrow, which contributes to an intraosseous hypertension and a decreased blood flow [3]. More and more evidences indicated that steroid increased in the number of adipocyte at the expense of osteoblast, leading to the insufficient bone remodeling and repairing of the necrotic bone $[4,5]$. Therefore, it is possible that the inhibition of marrow adipogenesis could provide a therapeutic target to prevent further increases in adipocyte formation with a resulting 
increase in functional bone cells [6]. Furthermore, it has been experimentally confirmed that lipid-lowering agent is able to prevent steroid-associated osteonecrosis [7,8].

Huogu (HG) formula, as a frequently used Chinese traditional herbal medicine, exhibits good effect in the treatment of osteonecrosis of the femoral head $(\mathrm{ONFH})$ in clinic applications with obvious advantages comparing to surgery [9]. Our previous studies reported that HG was effective in experimental ONFH $[10,11]$. Moreover, we further revealed that HG formula was able to regulate lipid metabolism by inhibition of peroxisome proliferators-activated receptor $(\mathrm{PPAR}) \mathrm{Y}$ and Wnt/ $\beta$-catenin signaling pathway in the ONFH rat model [12]. Interestingly, previous study reported that some lipid-lowering agents, such as Statins, could inhibit adipogenesis [8]. Given that Wnt/ $\beta$-catenin signaling pathway was the negative regulator of adipogenesis [13], we hypothesize that $\mathrm{HG}$ is able to inhibit adipogenesis via regulating $\mathrm{Wnt} / \beta$-catenin signaling pathway thus to delay the progression of ONFH.

The results of our preliminary experiment showed that the ethyl acetate fraction of HG (HGE), was the most effective fraction that accounted dominantly for its anti-adipogenic action (Suppl. Fig 1). To identify the main component of $\mathrm{HG}$, in the present study, we investigated the effect of HGE on adipogenic and osteogenic differentiation of rat BMSCs. Moreover, the possible mechanism associated with its effects on BMSCs differentiation was also explored.

Table 1. Composition of HG

\begin{tabular}{lll}
\hline Pharmaceutical name & Chinese Name & uses Amount (g) \\
\hline poria & Fuling & 12 \\
Cinnamomi ramulus & Guizhi & 10 \\
Atractylodis macrocephalae rhizoma & Baizhu & 12 \\
glycyrrhizae radix et rhizoma & Gancao & 3 \\
Pinelliae rhizoma praeparatum & Fabanxia & 9 \\
Radix Salviae Mittiorrhizae & Dangshen & 12 \\
Angelicae sinensis radix & Danggui & 9 \\
chuanxiongrhizoma & Chuanxiong & 10 \\
rehmanniae radix praeparata & Shudihuang & 12 \\
paeoniae radix rubra & Chishao & 9 \\
eupolyphagasteleophaga & Tubiechong & 9 \\
cervicornuscolla & Lujiaojiao & 12 \\
\hline
\end{tabular}

\section{Material and Methods}

\section{Reagents}

Dulbecco minimum essential medium (DMEM) high glucose, fetal bovine serum (FBS), L-glutamine, penicillin and streptomycin, were purchased from Invitrogen Life Technologies (Grand Island, NY, USA). Adipogenic induction medium (AIM) was provided by ScienCell (Carlsbad, CA, USA). All the primers used in this study were synthesized by Invitrogen Life Technologies. Oil Red O, Hoescht 33258, Dexamethasone, $\beta$-Glycerophosphate and ascorbic acid were provided by Sigma Alrich (Sigma Alrich, St Louis, MO, USA). Mouse anti-PPARY antibody was purchased from Santa Cruz Biotechnology (Santa Cruz, CA, USA), Rabbit anti-adipocyte protein2 (AP2) antibody was provided by Abcam (MA, USA), Rabbit anti-lipoprotein lipase (LPL) and rabbit anti-GAPDH antibody were obtained from Cell Signaling Technology (Beverly, MA, USA). CellTiter 96® AQueous One Solution Cell Proliferation Assay was obtained from Promega (Madison, WI, USA). BCIP/NBT ALP Color Development Kit was purchased from Beyotime (Shanghai, China). Enhanced chemiluminescence (ECL) reagent was provided by Pierce Technology (Pierce, USA).

\section{Animals}

Sprague-Dawley (SD) rats (4-7-week-old) were housed in Laboratory Animal Center (China Academy of Chinese Medical Sciences, Beijing, China) according to the guidelines for the care and use of laboratory animals of the NIH and China Academy of Chinese Medical Sciences (Beijing, China). All experimental procedures were approved by the Committee for Animal Use of the China Academy of Chinese Medical Sciences.

\section{Preparation of HGE}

HG formula is composed of 12 traditional herbs as indicated in Table 1. The Chinese herbal medicines were purchased from Tong-Ren-Tang Ltd. (Beijing, China) and identified by Professor Zhang Cun (Institute of Chinese Materia Medica, China Academy of Chinese Medical Sciences, Beijing, China). The voucher specimens (No. HG20120901-12) have been deposited in our laboratory. All the drugs conformed with the requirement of China pharmacopoeia [14]. According to the prescription ratio of HG formula, 11 drugs except Cervi Cornus Colla were boiled in water for 2 times [1:10(w/v) 
for $1 \mathrm{~h}$ each time], then filtered and condensed. The concentrated water solution was deposited by adding $95 \%$ ethanol to make the whole solution as $60 \%$ ethanol solution. Cervi Cornus Colla was dissolved in hot water and merged into the above solution. After $48 \mathrm{~h}\left(\right.$ at $\left.4^{\circ} \mathrm{C}\right)$, the supernatant liquor was centrifuged, and condensed in vacuum to proper amount. The final concentration of $\mathrm{HG}$ is $1.91 \mathrm{~g}$ (crude herbs)/g liquid extract. $50 \mathrm{~g}$ of the liquid extract of was dissolved in $100 \mathrm{~mL} \mathrm{H}_{2} \mathrm{O}$ and extracted with four different solvent successively. Each combined solution and the remaining water solution were concentrated in vacuum to gain five separated parts as aqueous fraction (HGA, 29.38g), petroleum ether fraction (HGP, 0.26g), chloroform fraction (HGC, $0.08 \mathrm{~g}$ ), ethyl acetate fraction (HGE, 0.12g), and $\mathrm{n}$-butanol fraction (HGB, $1.14 \mathrm{~g}$ ) respectively. The flow chart is shown in Fig 1 . All the fractions except HGP for the poor solubility were screened in the following osteogenesis and adipogenesis experiment.

\section{Qualitative HPLC analysis}

The ethyl acetate extract was diluted with distilled water to the proper concentration before use as following. For HPLC analysis, ethyl acetate extract was dissolved in methanol to $0.012 \mathrm{~g} / \mathrm{mL}$. The representative compounds were set as standards according to the guideline of China pharmacopoeia [14]. Standards of 5-hydroxymethylfurfural, chlorogenic acid, albiflorin, paeoniflorin, ferulic acid, liquiritin, acteoside, ligustilide, cinnamaldehyde, lobetyolin, cinnamic acid, and benzoylpaeoniflorin were purchased from the National Institute for Food and Drug Control (Beijing, China). The purity of all compounds was more than $98 \%$ (determined by HPLC). They were prepared at a proper concentration in methanol. Methanol and acetonitrile (HPLC grade, Fisher Scientific, New Jersey, USA), and pure water (Wahaha Group Co., Ltd., China) were used for HPLC analysis. All other chemicals were of analytical grade. The solutions were filtered through $0.22 \mu \mathrm{m}$ membranes before HPLC analysis.

HPLC was performed on a Shimadzu HPLC system (Shimadzu Corporation, Japan) equipped with an LC-20AT binary pump, an SPD-M20A diode array detector, a CBM-20 Alite system controller, a SIL-20A auto-sampler, a DGU-20A5 degasser, and a CTO-10ASvp column oven. An Agilent TC(2) C18 column $(250 \times 4.6 \mathrm{~mm}, 5 \mu \mathrm{m})$ was maintained at $35^{\circ} \mathrm{C}$. Detection wavelength was set at $254 \mathrm{~nm}$. The mobile phase for fingerprint analysis consisted of methanol (A) and $0.1 \%$ formic acid (B) at a flow-rate of 1.0 $\mathrm{mL}$ min-1, A gradient program was used as follows: 0-40min-70min-80min, $5 \%-32 \%-66 \%-100 \%$, the injected volume was $10 \mu \mathrm{l}$. Online UV spectra were recorded in the range of $190-800 \mathrm{~nm}$.

\section{Bone marrow stromal cells isolation and culture}

BMSCs were isolated from male rats as previously described $[15,16]$. Briefly, BMSCs were obtained from the femurs and tibias by flushing with $5 \mathrm{ml}$ complete medium DMEM-HG with 10\% FBS and $1 \%$ penicillin/streptomycin and incubated at $37^{\circ} \mathrm{C}$ with $5 \%$ CO2. Cells from the 4 th passage were used in the subsequent assays.

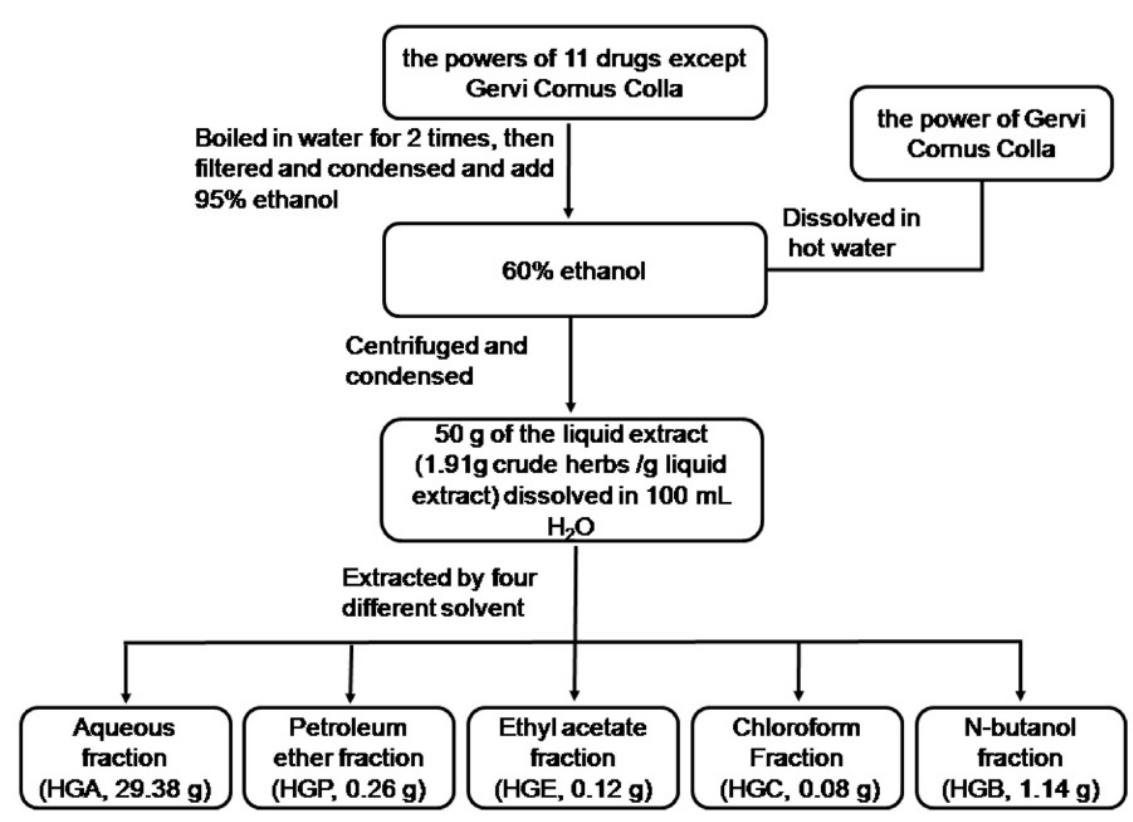

Figure 1. Flow chart representative of the extraction processes of HG.

\section{Osteogenic and adipogenic differentiation}

BMSCs were seeded as a monolayer in $25 \mathrm{~cm}_{2}$ flask. For osteogenic differentiation, BMSCs were cultured in normal growth medium until $50 \%$ confluent, and osteogenic differentiation was induced by osteogenic induction medium (OIM), that is DMEM with $0.1 \mu \mathrm{M}$ dexamethasone, $10 \mathrm{mM}$ $\beta$-Glycerophosphate, $50 \mathrm{mg} / \mathrm{L}$ ascorbic acid and 10\% FBS. Adipogenic differentiation was achieved by using induction in the AIM according to the manufacturer's instructions. The medium was changed every 2-3 days and the culture was maintained for 7 to 21 days for different assays. 


\section{ALP staining and quantification assay}

After BMSCs were incubated with OIM in the presence of HGA, HGE, HGC, HGB or the vehicle control for 7 days, cells were washed with PBS and fixed with $10 \%$ formalin for $15 \mathrm{~min}$. Fixed cells were subjected to an assay for ALP activity by the BCIP/NBT Alkaline Phosphatase Color Development Kit according to the manufacturer's protocol (Beyotime, Shanghai, China). The images were taken with a digital camera attached to the microscope. Image-Pro Plus software version 6.0 (IPP6.0, Media Cybernetics, Inc., MD) was used to calculate the ALP activity indicated as the integrated optical density (OD) of staining in unit culture area. Then alkaline phosphatase buffer was added and quantified using microplate reader (Bio-Rad, USA) at $405 \mathrm{~nm}$. All procedures were conducted in triplicate.

\section{Oil Red $O$ staining and quantification assay}

After BMSCs were incubated with AIM in the presence of HGA, HGE, HGC, HGB, Sim (as positive control) or the vehicle control for 14 days, cells were fixed with $10 \%$ formalin for $15 \mathrm{~min}$. Followed by washing with $50 \%$ ethanol cells and were stained with $0.2 \%$ Oil Red O (Sigma Alrich, St Louis, MO, USA) solution for $20 \mathrm{~min}$ at room temperature. Then washed the Oil Red O stained cells with PBS, the extraction of the Oil Red $\mathrm{O}$ dye was performed by using extraction buffer ( $50 \% \mathrm{EtOH}, 2 \%$ (w/v) SDS) for $15 \mathrm{~min}$ at room temperature. Quantification of the Oil Red O dye was performed at OD 595 using an ELISA reader (Bio-Rad, USA). For the inhibitor experiments, BMSCs were induced to adipocytes differentiation with $0.2 \mu \mathrm{g} / \mathrm{ml}$ of DKK-1(R\&D system Inc. MN, USA) or $2 \mathrm{ng} / \mathrm{ml}$ of Noggin (Sigma Alrich, St Louis, MO, USA). All tests were conducted in triplicate.

\section{Cell survival viability assay and apoptosis analysis}

Cell viability was determined by 3-(4,5-dimethyl-2-thiazolyl)-2,5-diphenyl-2H-tetrazoli um bromide (MTS) method using CellTiter 96® AQueous One Solution Cell Proliferation Assay (Promega, USA) according to the manufacturer's instructions. And the BMSCs proliferation was detected by Cell Proliferation ELISA BrdU (colorimetric) kit (Sigma Alrich, St Louis, MO, USA) according to the manufacturer's instructions. The experiments were carried out 3 times in triplicate measurements.

Detection of BMSCs apoptosis was performed by Hoechst 33258 staining as described previously [17]. The BMSCs were incubated with different concentration of HGE $(1,10,100 \mathrm{ng} / \mathrm{mL})$ for $48 \mathrm{~h}$, following with fixation with $4 \%$ formaldehyde for
$15 \mathrm{~min}$ at room temperature, then stained with Hoescht 33258 for $15 \mathrm{~min}$. Cells with nuclei containing condensed chromatin or cells with fragmented nuclei were defined as apoptotic cells by visualization. Apoptosis was quantified by the percent of apoptosis cells to control cells. The experiments were carried out 3 times in triplicate measurements.

\section{RNA-isolation and quantitative RT-PCR}

Total RNA was isolated from cultured BMSCs by using TRIzol Reagent. cDNA was synthesized from extracted RNA using Taq-Man RT reagents according to the manufacturer's instructions. SYBR green I was used to monitor amplification of DNA on the Cycler thermal cycler and real-time quantitative PCR detection system (Bio-Rad, CA, USA). The primers sequences for AP2, LPL and PPAR $\gamma$ are listed in Table 2. GAPDH was utilized as an internal control to normalize for differences in the amount of total RNA in each sample. DNA amplification was conducted by an ABI Prism 7500 Real-time PCR System (Applied Biosystems, Foster City, CA). The experiments were carried out 3 times in triplicate measurements.

\section{Western blotting}

After BMSCs were cultured with or without AIM and $\operatorname{HGE}(1,10,100 \mathrm{ng} / \mathrm{mL})$ for $14 \mathrm{~d}$, cell pellets were lysed in RIPA lysis buffer (50 mM Tris- $\mathrm{HCl}$, pH 7.4, $300 \mathrm{mM} \mathrm{NaCl}, 5 \mathrm{mM}$ EDTA, $0.5 \%$ Triton X-100, $1 \mathrm{mM}$ $\mathrm{NaF}, 1 \mathrm{mM}$ Na3VO4, and protein inhibitor cocktail) for $30 \mathrm{~min}$ on ice. Lysates were centrifuged at 12,000 $\mathrm{g}$ for $20 \mathrm{~min}$ at $4^{\circ} \mathrm{C}$. Eighty microgram of protein was separated on $10 \%$ SDS-PAGE gels and then electro transferred onto a PVDF transfer membrane. The membranes were immunoblotted with primary antibody for AP2, LPL and PPAR $\gamma$ in TBST containing $1 \%$ BSA in overnight at $4{ }^{\circ} \mathrm{C}$ after blocked in $5 \%$ non-fat dry milk in TBS. Horseradish peroxidase (HRP)-conjugated anti-mouse and anti-rabbit antibodies (1:5000) were used as secondary antibodies. The membranes were then visualized using the ECL system. GAPDH as internal control was used to confirm equal protein loading.

\section{Experimental model of steroid-induced osteonecrosis of the femoral head on rats}

Adult male SD rats (specific pathogen-Free, Certificate no. SCXK (JUN) 2016-0004), weighing 220-250 g were used in the study. Steroid-induced ONFH rat models were constructed as our previously reported [12]. Briefly, rats were injected subcutaneously at the back by methylprednisolone acetate (MPSL; Pfizer Manufacturing BeLgium NV, Belgium) at the dosage of $21 \mathrm{mg} / \mathrm{kg}$ daily for $3 \mathrm{~d}$. Those received HGE $(3 \mathrm{mg} / \mathrm{kg}$ or $6 \mathrm{mg} / \mathrm{kg}$ ) by 
intragastric administration were set as HGE-treated groups. The rats received distilled water with or without MPSL were considered as model or normal group, separately. The left femora were obtained and fixed for three days in $4 \%$ paraformaldehyde ( $\mathrm{pH7.4}$ ) and then decalcified with 10\% EDTA, then sectioned for haematoxylin and eosin staining. The right femoral head tissues were dissected from rats were homogenized and total proteins were extracted for western blot to detect the expression of Wnt, BMP2 and PPAR $\gamma$ by specific antibodies.

\section{Statistical Analysis}

All raw data were processed by authorized software SPSS 13.0. The data were analyzed by One-way ANOVA followed by Dunnett's t-test to assess the statistical significance of the differences between the study groups. Differences were considered statistically significant when $P$ was less than 0.05 .

\section{Results}

\section{Chemical composition of HGE}

To investigate the effective components in HG, we performed extraction by using different solvents as described in Methods and analyzed each extract by HPLC. The HPLC chromatogram of HGE in Fig. 2 is the representative of these extracts according to the literature [14]. By comparison of the retention times and on-line UV spectra of the reference standards, a total of 12 compounds were identified. According to the chemical investigations of HG, acteoside and 5-hydroxymethylfurfural were attributed to rehmanniae radix praeparata. Albiflorin, paeoniflorin and benzoylpaeoniflorin were owed to paeoniae radix rubra. Ferulic and ligustilide were from angelicae sinensis radix and chuanxiong rhizome. Liquiritin was obviously belonged to glycyrrhizae radix et rhizoma. Cinnamaldehyde and cinnamic acid were originated to cinnamomi ramulus, and lobetyolin was ascribed to Radix salvia Miltiorrhizae. Base on the relative peak area, chlorogenic acid, ferulic acid, ligustilide, lobetyolin, cinnamicacid and benzoylpaeoniflorin apparently were the main components of HGE.

\section{HGE significantly attenuated the BMSCs adipogenic differentiation and slightly promoted osteogenic differentiation in the in vitro culture system}

BMSCs are pluripotent cells that have the capacity to differentiate into osteoblasts and adipocytes in the niche of marrow. To determine the main effective components of $\mathrm{HG}$, we previously screened the effect of the HG fractions on the differentiation of BMSCs. Interestingly we found that HGE effectively inhibited adipogenesis whereas HGA promoted the formation of osteocytes obviously. In this study we further investigated the effect of HGE on adipogenic differentiation of BMSCs. As shown in Fig. 3A, AIM, a commonly used inducer for adipogenic differentiation in BMSCs, could obviously induce adipogenic differentiation, as demonstrated by accumulation of lipid droplets in the cytoplasm. However, Adipogenic differentiation of BMSCs was inhibited in the presence of HGE with a dose-dependent manner. The quantitative result showed that adipogenic differentiation of BMSCs could be inhibited in nearly $60 \%$ at the concentration of $100 \mathrm{ng} / \mathrm{ml}$ of HGE (Fig 3B-C), a similar inhibitory capability as simvastatin (Sim) at $1 \mu \mathrm{M}$.

It has been demonstrated that there is a reciprocal relationship in the differentiation of adipocytes and osteoblasts from BMSCs of the bone marrow [18]. To determine the effect of HGE on osteogenic differentiation from rat BMSCs, the cells were induced in OIM with or without HGE for up to 7 days and evaluated by Alkaline Phosphatase (ALP) staining after termination of the induction. As indicated in Fig 4, HGE could slightly promote osteogenic differentiation at the concentration of 1-100 $\mathrm{ng} / \mathrm{mL}(P<0.05$, Fig $4 \mathrm{~A}$ and $\mathrm{B})$.

Table 2. Primers sequence for real-time quantitative PCR assays

\begin{tabular}{ccccc}
\hline Gene & Accession number & Forward (5'-3') & Reverse (5'-3') & $\begin{array}{c}\text { Product } \\
\text { size(bp) }\end{array}$ \\
\hline LPL & NM_012598.2 & GGAGGTCGCCACAAATAAAA & CTGACCAGCGGAAGTAGGAG & 113 \\
AP2 & NM_053365.1 & ATGTGTCATGAAAGGCGTGA & AAACCACCAAATCCCATCAA & 119 \\
PPARY & NM_013124.3 & CATITITCAAGGGTGCCAGT & GAGGCCAGCATGGTGTAGAT & 156 \\
GAPDH & XM_017593963.1 & AGACAGCCGCATCTTCTTGT & CTTGCCGTGGGTAGAGTCAT & 207 \\
\hline
\end{tabular}




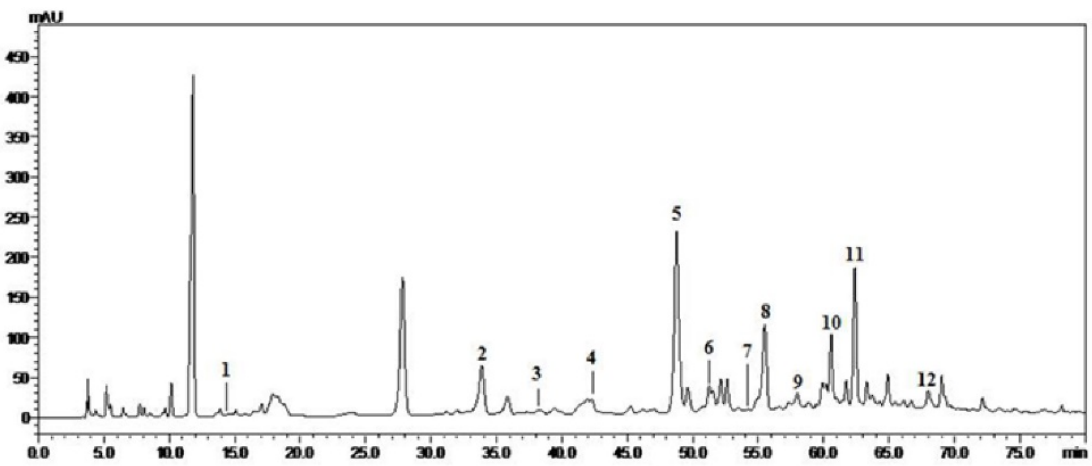

Figure 2. HPLC profile and the identified compounds from HGE (UV chromatograms at $254 \mathrm{~nm}$ ). 1: 5-hydroxymethylfurfural. 2: chlorogenicacid. 3: albiflorin. 4: paeoniflorin. 5: ferulicacid. 6: liquiritin. 7:acteoside. 8: ligustilide. 9: cinnamaldehyde. 10: lobetyolin. 11: cinnamicacid. 12: benzoylpaeoniflorin.
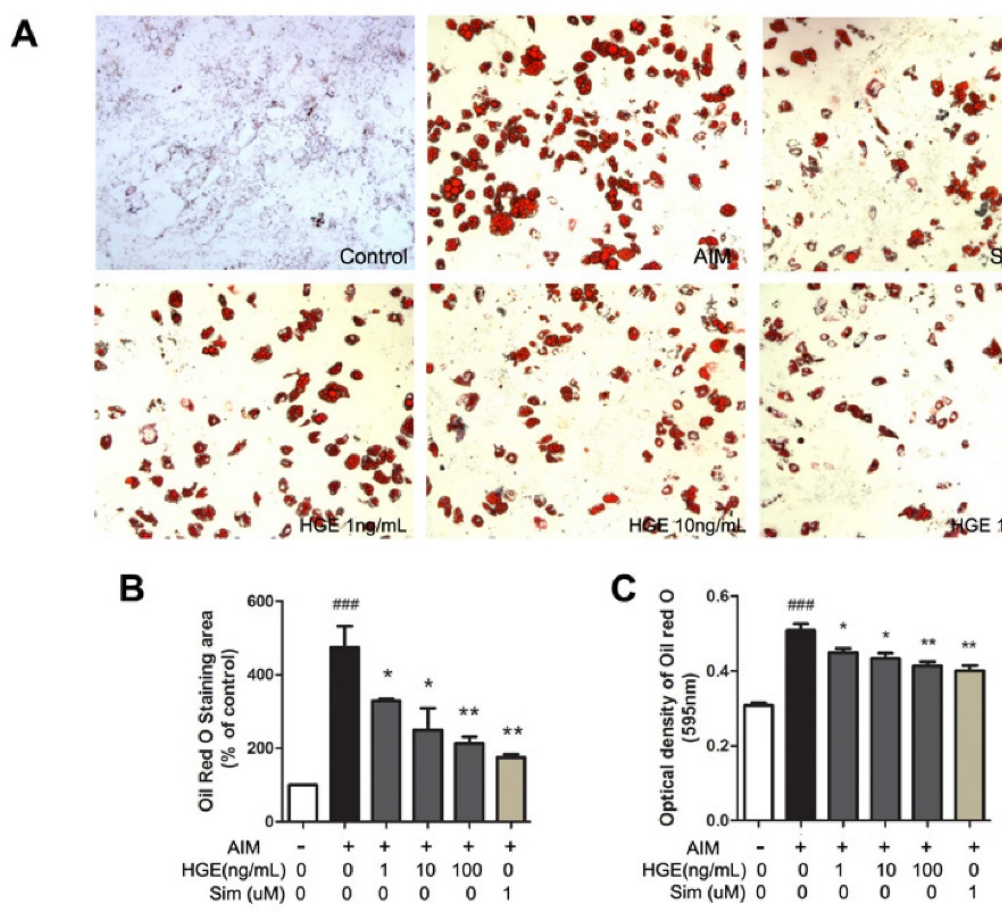

Figure 3. HGE inhibits the adipogenesis of BMSCs by Oil Red O staining. (A) BMSCs were stained with Oil-red $\mathrm{O}$ staining after the cells were incubated with $\mathrm{HGE}$ $(1,10,100 \mathrm{ng} / \mathrm{mL})$ in AIM for 14 days. The images shown are representative of 3 independent experiments. Adipogenesis activity was quantified by percentage of Oil-red stained positive area compared to control (B) or optical density tested by microplate reader at $595 \mathrm{~nm}$ by using extraction buffer (C). Data are represented as the mean $\pm \mathrm{SD}$. \#\#P 0.001 compared with Control; $* P<0.05, * * P<0.01$, compared with AIM.

To rule out the anti-adipogenic effect of HGE was due to reduced cell viability, MTS assays were carried out. As shown in Fig. 4C, HGE did not show any suppressive effect on the viability on BMSCs at the concentrations from 1 to $100 \mathrm{ng} / \mathrm{mL}$ after treatment for $72 \mathrm{~h}$. On the contrary, HGE promoted the proliferation of BMSCs at a higher concentration (Fig. 4D). Meanwhile HGE did not show any affect on the colorimetric assays (Suppl Fig 2). Furthermore, apoptotic death of BMSCs was not detectable under the condition (Fig. $4 \mathrm{E}$ and F). Therefore, it is likely that HGE specifically suppressed adipogenic differentiation of BMSCs.

\section{HGE inhibited the adipogenic gene expression of the cultured BMSCs}

To determine the molecular mechanism by which HGE inhibited adipogenic differentiation of BMSCs, we examined the expression of adipocyte-specific genes such as PPARY, LPL and AP2 in the cells after induction by AIM in the presence or absence of HGE. The expression of PPAR $\gamma$, LPL, and AP2 genes in the cells was analyzed by real-time PCR. Obviously AIM treatment significantly up-regulated the level of these genes (Fig. 5). As expected, the presence of HGE resulted in markedly reduced expression of PPAR $\gamma$, LPL, and AP2 with a dose-dependent manner, suggesting that the inhibitory effect of HGE on adipogenic 
differentiation was through suppressing the expression of the adipogenic genes.

\section{Involvement of the BMP and Wnt pathways for the anti-adipogenic effect of HGE}

The role of the BMP and Wnt-signaling pathways in mediating adipogenesis of BMSCs has been well established. The BMP pathway stimulates adipogenesis, while the Wnt-signaling pathway inhibits adipogenesis [19]. To determine the effect of HGE on both pathways, we compared the effect of the inhibitors for the BMP and Wnt pathways on the expression of marker proteins affected by HGE in the

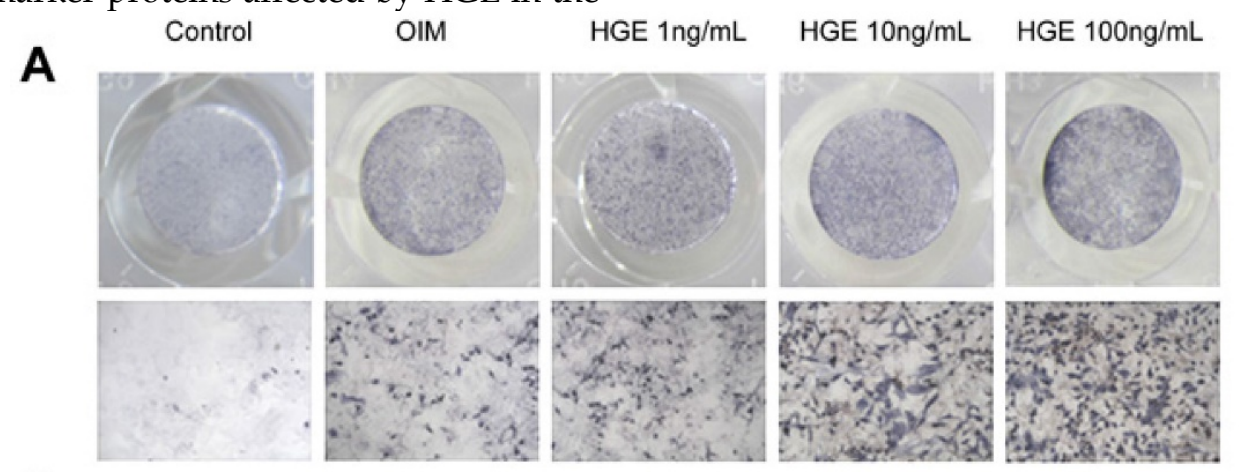

B
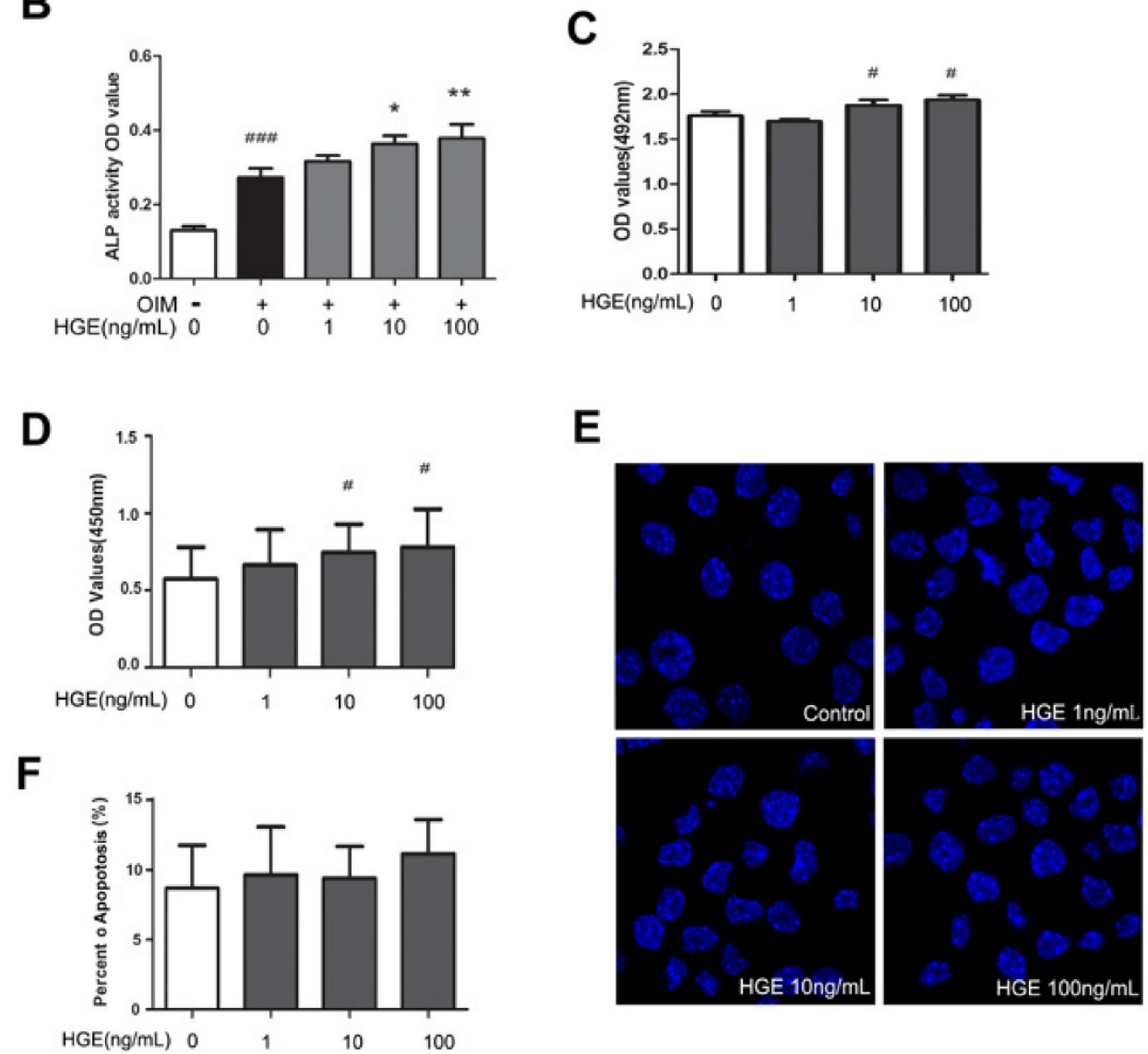

adipogenic differentiation. As shown in Fig 6 A-D, the protein expression of PPARY, LPL, and AP2, the main transcription factors during the differentiation of BMSCs into adipocytes, was suppressed by HGE significantly. Interestingly, DKK-1, an inhibitor of Wnt, not Noggin, an inhibitor of BMPs, could restore the expression of these factors suppressed by HGE in the presence of AIM, suggesting that HGE may directly impact the Wnt signaling pathway. On the contrary, Inhibition of the BMPs and HGE synergistically suppress the product of these genes.

Figure 4. HGE slightly promotes osteogenic differentiation in BMSCs without affecting the cell viability and apoptosis. (A) BMSCs were treated with HGE (1, 10, 100 $\mathrm{ng} / \mathrm{mL}$ ) in OIM for 7 days, then evaluated by ALP staining. The images shown are representative of three independent experiments. (B) ALP activity was quantified by optical density tested by microplate reader at $405 \mathrm{~nm}$ by incubated with alkaline phosphatase buffer. The effects of HGE on the viability of BMSCs for $72 \mathrm{~h}$ were tested by MTS (C) or Cell Proliferation by BrdU (colorimetric) method (D). (E) BMSCs were cultured with HGE for $48 \mathrm{~h}$ and stained with Hoescht 33258 . Cells with nuclei containing condensed chromatin or cells with fragmented nuclei were defined as apoptotic cells (Magnification 600x). (F) Apoptosis was quantified by the percent of apoptosis cells to control cells. Data are represented as the mean $\pm \mathrm{SD}$. $\# P<0.05, \ldots+1 \mathrm{P}<0.001$ compared with Control; $* P<0.05$, $* * P<0.01$, compared with $\mathrm{OIM}$. 

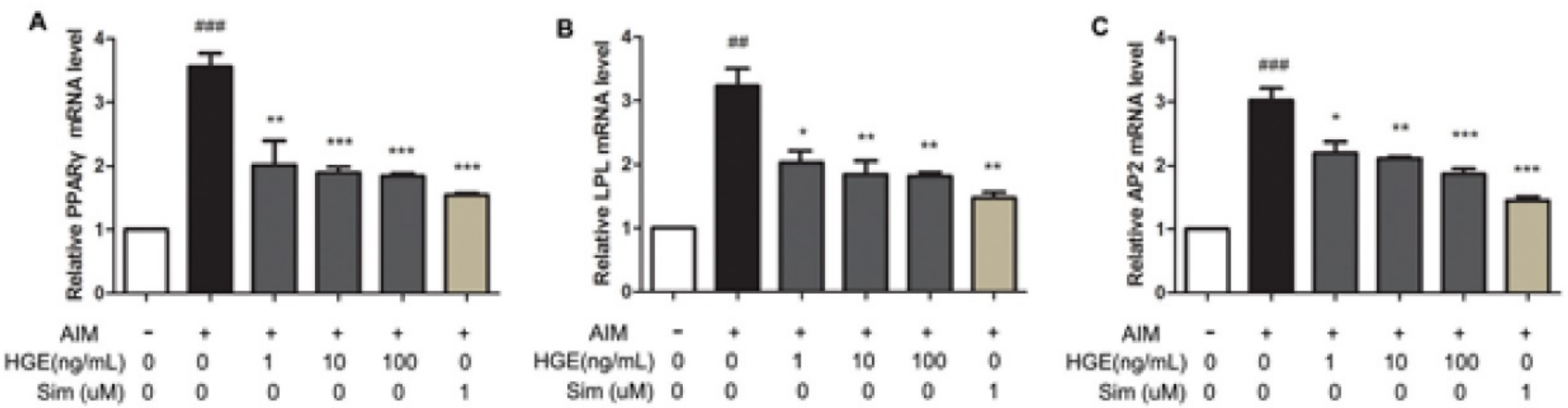

Figure 5. HGE suppresses expression levels of PPAR $\gamma, \mathrm{LPL}$, and AP2 mRNA in BMSCs. BMSCs were incubated with HGE (1, 10,100 ng/mL) in AIM for 7 days, then gene expressions were detected by real-time PCR. Data are represented as the mean \pm SD. $\# P<0.01, \ldots \# P<0.001$ compared with Control; $* P<0.05$, $* * P<0.01$ compared with AIM.

A
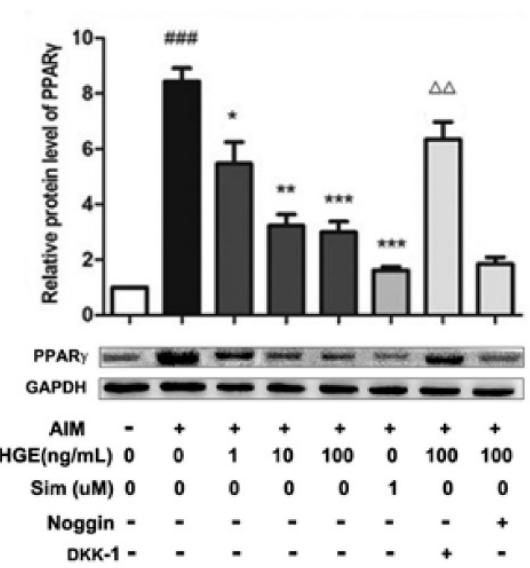

B
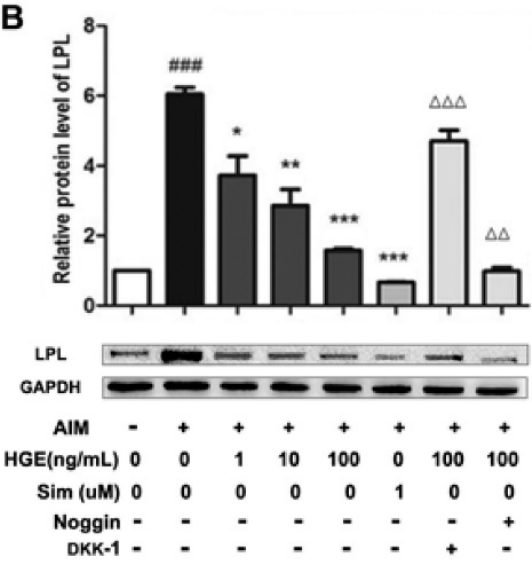

C
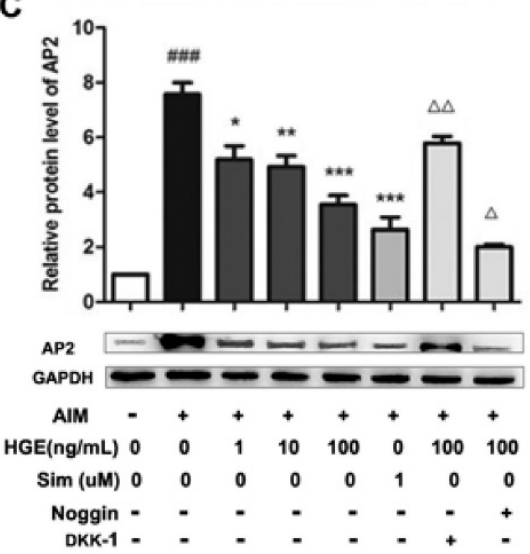

Figure 6. Noggin enhances while DKK-1 restores HGE-mediated regulation of adipogenic proteins. BMSCs were exposed to HGE (100 ng/mL) and/or Noggin (BMP inhibitor) or DKK-1 (Wnt inhibitor) for 14 days, then cell lysates were subjected to Western blotting for PPAR, LPL, and AP2. Relative amount of protein were determined by densitometric analysis for PPARY (A), LPL (B), and AP2(C). The results shown are representative of three independent experiments. Data are

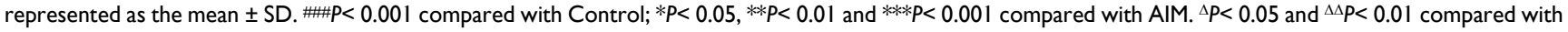
HGE $(100 \mathrm{ng} / \mathrm{mL})$.

To further demonstrate the involvement of the signaling pathways in the anti-adipogenic effect of HGE, lipid droplets in BMSCs treated with HGE were detected by oil red $\mathrm{O}$ staining in the presence or absence of the chemical inhibitors against BMPs and Wnt. As shown in Fig. 7, lipid accumulation suppressed by HGE could be restored by adding DKK-1, not Noggin. HGE and Noggin synergistically promoted adipogenesis in the cells. Taken together, our results suggested that HGE suppressed adipogenesis of BMSCs through, at least in part, regulating the BMP and Wnt-signaling pathways.

\section{HGE inhibits the bone marrow fat formation in steroid-induced ONFH rats}

Previous studies reported that steroid caused significant enlargement of the adipocyte size and fat accumulation in the bone marrow, which contributes to the onset and progress of ONFH [20, 21]. To investigate the role of HGE in repairing osteocytes damage, we performed steroid-induced ONFH rat model, which resulted in a significant osteonecrosis and extensive fat accumulation in the bone marrow, and allowed the rats to be recovered under the conditions with or without HGE treatment. As shown in Fig 8A and B, the ratio of empty lacunae in the bone trabeculae of the model group was significantly increased compared with that of control group. However, rats in the HGE-treated groups had much lower empty lacunae ratio than that in the ONFH group alone $(P<0.05$ or $P<0.01)$. Moreover, the adipose area and adipocyte perimeter in the bone marrow, which were dramatically elevated in steroid-induced ONFH rats, were both significantly reduced by HGE treatment (Fig $8 \mathrm{~A}, \mathrm{C}$ and D). In addition, HGE significantly increased the expression of Wnt3a, in contrast markedly decreased the expression of PPAR $\gamma$, while has no obvious effect on BMP2 (Fig 8 E-G). Taken together, the results demonstrate that HGE is able to reduce fat formation and repair the osteocytes damage caused by steroid. 
A
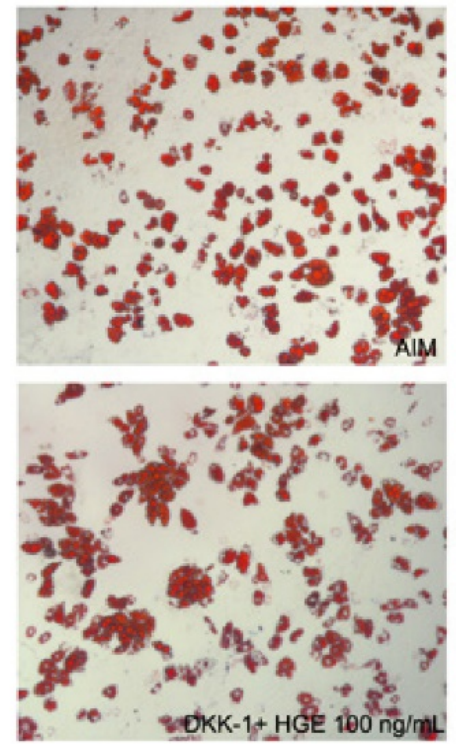
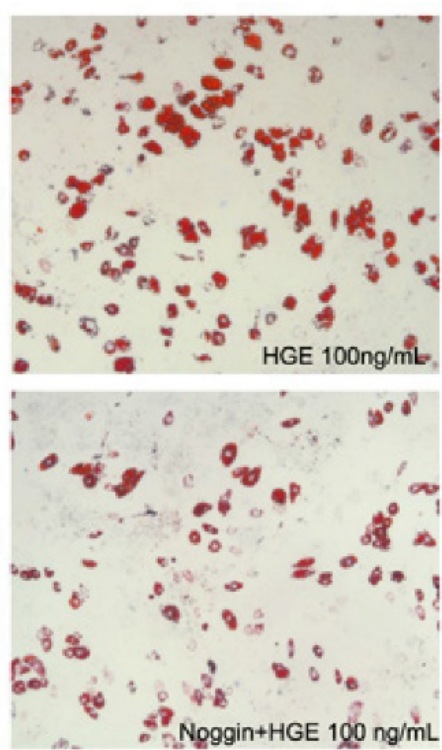

B

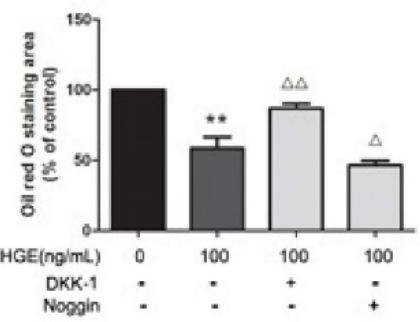

C

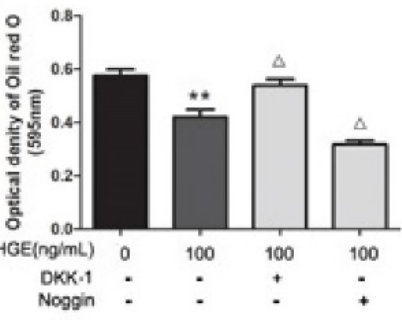

Figure 7. Involvement of BMP and Wnt pathways in the anti-adipogenic effect of HGE. (A) BMSCs were exposed to HGE (100 ng/mL) and/or Noggin (BMP inhibitor) or DKK-1 (Wnt inhibitor) for 14 days, then the adipogenesis of BMSCs were stained by Oil-red O staining and quantified by the quantified by percentage of Oil-red stained positive area compared to control (B) or optical density tested by microplate reader at $595 \mathrm{~nm}$ by using extraction buffer (C). The results shown are representative of 3 independent experiments. Data are represented as the mean $\pm \mathrm{SD}$. $* * P<0.01$ compared with AIM. ${ }^{\Delta P}<0.05$ and ${ }^{\Delta \Delta} P<0.01$ compared with $\mathrm{HGE}$ $(100 \mathrm{ng} / \mathrm{mL})$.

A

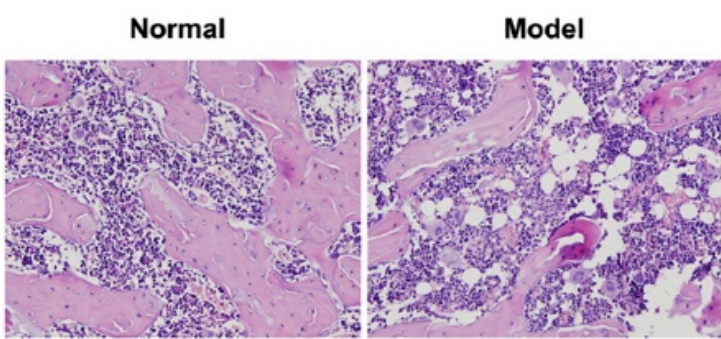

HGE $3 \mathbf{~ m g / k g}$

HGE $6 \mathrm{mg} / \mathrm{kg}$

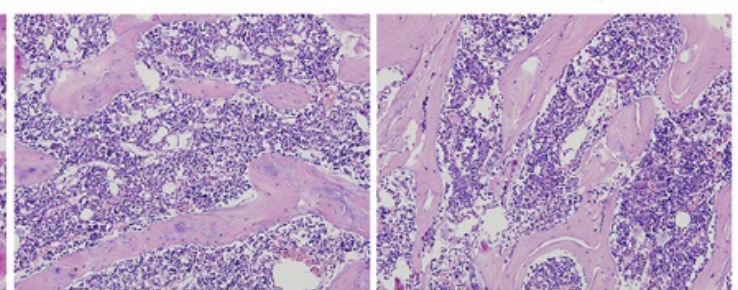

B

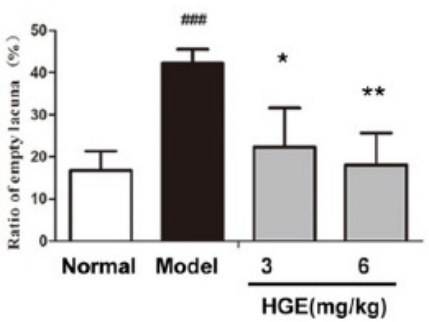

E

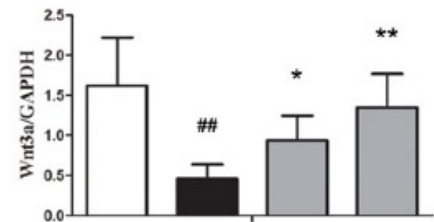

Wnt3a

GAPDH

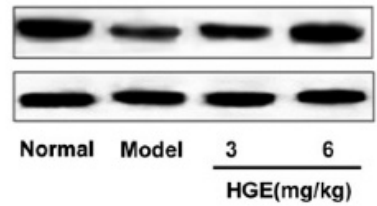

C

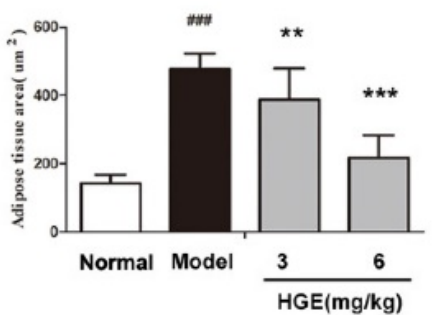

F

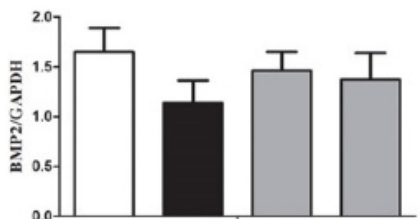

BMP2

GAPDH

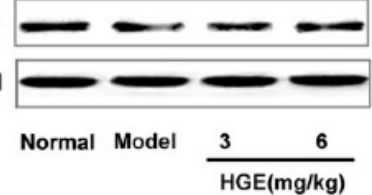

D

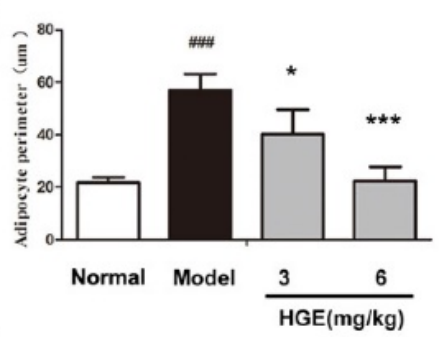

G

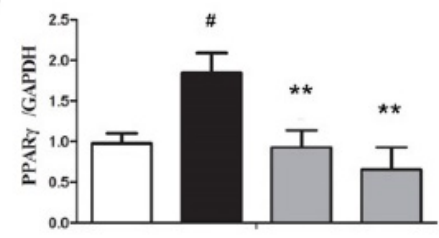

PPAR $\gamma$

GAPDH

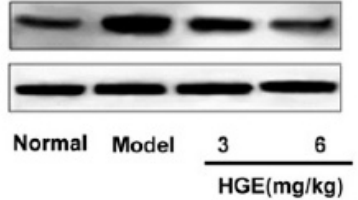

Figure 8. HGE inhibits adipogenesis in steroid-induced ONFH rats. (A) Hematoxylin and eosin staining photomicrographs of formal head from normal, vehicle and HGE-treated steroid-induced ONFH rats, respectively. Statistical analysis of the ratio of empty lacuna (B), the adipose tissue area (C), and the adipocyte perimeter (D). Protein expressions of Wnt3a (E), BMP2 (F) and PPAR $\gamma(G)$ in the femoral heads of steroid-induced ONFH rats with or without HGE treatment were detected by western blot, respectively. Data are represented as the mean $\pm S D(n=12$ for each group.). $0.05, * * P<0.01$ and ${ }^{* * *} P<0.001$ compared with the Model group. 


\section{Discussion}

HG is a clinical effective prescription drug used for treatment of non-traumatic ONFH in China [9]. Our previous studies revealed that HG is able to improve the lipid metabolism disorders on the steroid-induced osteonecrosis of the femoral head rats. Especially the adipose tissue area and the adipocyte perimeter in the bone marrow were dramatically reduced by HG. In the present study, we examined the effect of HGE, the ethyl acetate extract of $\mathrm{HG}$, on adipogenic and osteogenic differentiation in rat primary BMSCs and in the steroid-induced ONFH rat. The results indicated that HGE significantly inhibited adipogenic differentiation, but slightly promoted osteogenic differentiation of BMSCs through regulating the BMP and Wnt signaling pathway. These findings expanded our horizon of understanding the molecular mechanism underlying the role of $\mathrm{HG}$ in prevention of ONFH.

Elevated adipogenesis of BMSCs is closely associated with steroid-induced osteonecrosis [22, 23]. Adipocytic and osteogenic cells are believed to be derived from multi-potential stromal cells in the marrow, and a large body of experimental evidence suggests that an inverse correlation exists between adipogenesis and osteogenesis, because differentiation towards an adipocytic phenotype occurs at the expense of an osteoblast phenotype [24]. It has been reported that culture supplements up-regulate adipogenic differentiation with associated down-regulation of osteogenic differentiation or vice versa $[13,25,26]$. Besides having a passive role of space filling in the bone marrow cavity, medullary adipocytes also function as secretory cells to influence osteogenesis and hematopoiesis [27-29]. The accumulation of fat cells and/ or their secreted molecules such as leptin and adiponectin in the bone marrow has been shown to affect several aspects of bone remodeling, including the development of osteoclasts, synthesis of RANKL and OPG, an antagonist of RANKL, and the tight coupling of cellular signaling between osteoblasts and osteoclasts [30]. Thus, reversal of adipogenesis may be of importance as a therapeutic approach to treat ONFH. Our findings reveal that HGE could markedly inhibited adipogenic differentiation of BMSCs on the cultured system and in the steroid-induced ONFH rat model, suggesting that the therapeutic effectiveness of HG in the clinic may be through suppressing adipogenesis.

A variety of the signaling pathways lead to the adipogenic and/ or osteogenic differentiation of BMSCs. PPAR $\gamma$ are believed to be the master regulator of adipogenesis and also show well described anti-osteoblastogenic effects. PPAR $\gamma$ deficient embryonic stem cells failed to differentiate into adipocytes, instead they differentiated into osteoblasts [31]. TZD rosiglitazone and 15-deoxy-delta $(12,14)-P G J 2$, two commonly used agonists of PPAR $\gamma$, promote BMSCs adipogenesis, but inhibit osteogenesis [32]. In this study, we found that HGE was able to inhibit the expression of PPAR $\gamma$, LPL and AP2, adipogenic marker genes, both at the mRNA and protein levels, which confirmed the morphological observation by Oil Red $\mathrm{O}$ staining. Furthermore, HGE could inhibit the expression of PPAR $\gamma$ in the steroid-induced ONFH rat model.

The inverse correlation of adipogenic and osteogenic lineage differentiation in BMSCs is mediated by diverse signaling pathways. Some of these factors play an opposite role with respect to lineage determination, while others function in a complementary fashion. For example, the activation of the Wnt signaling pathway has shown to promote osteogenesis while it also represses adipogenesis [13, 33]. In contrast, BMPs signaling as BMP-2, BMP-4, and BMP-9 can stimulate both the adipogenic and osteogenic pathways depending on the activated specific receptor [34, 35]. Our results showed that HGE significantly decreased the adipocyte differentiation, while slightly increased the ALP activity in the in vitro experiment system. Furthermore, results from the steroid-induced ONFH rat model displayed that HGE could up-regulate the expression of Wnt, while slightly increase the expression of BMP2 without statistical significance, which indicated that HGE could coordinately regulate both the Wnt and BMPs signaling pathways.

To further confirm the involvement of the Wnt and BMPs signaling pathways in the effect of HGE on adipogenic lineage differentiation, the pharmacological inhibitors for these two pathways were applied in our experiments. Our results demonstrated that HGE significantly reduced the adipogenic master gene PPAR $\gamma, \mathrm{LPL}$ and AP2 expression in a dose-dependent manner. However, addition of DKK-1, a Wnt inhibitor, could efficiently restore the inhibitory effect of HGE on the expression of PPAR $\gamma, \mathrm{LPL}$ and AP2 in BMSCs, suggesting that HGE inhibited adipogenesis through activating the Wnt pathway. Interestingly, the expression of PPAR $\gamma$, LPL and AP2 was synergistically suppressed by addition of noggin, a BMP inhibitor, implicating that the BMP signal pathway also contributed to the inhibition of adipogenesis by HGE. As to the promotion effect of HGE on osteogenesis, it suggested that he may more effectively impact on the Wnt pathway than inhibition of BMP. Taken together, our data demonstrate that the Wnt and BMP pathways 
mediate the role of HGE in anti-adipogenic differentiation of BMSCs, which summarized in Fig 9.

Conclusively, we have demonstrated that HGE treatment significantly inhibited adipogenesis and slightly promoted osteogenesis of BMSCs due to effectively regulation of BMP and Wnt pathways both in vitro and in vivo. Our findings shed lights on the action and mechanism of HGE on the inhibition of adipogenesis and provide scientific rationale for using HGE in the treatment of ONFH. Further, this study also supports potential role of HGE as a novel agent to prevent disorders involving excessive adipogenesis of BMSCs.

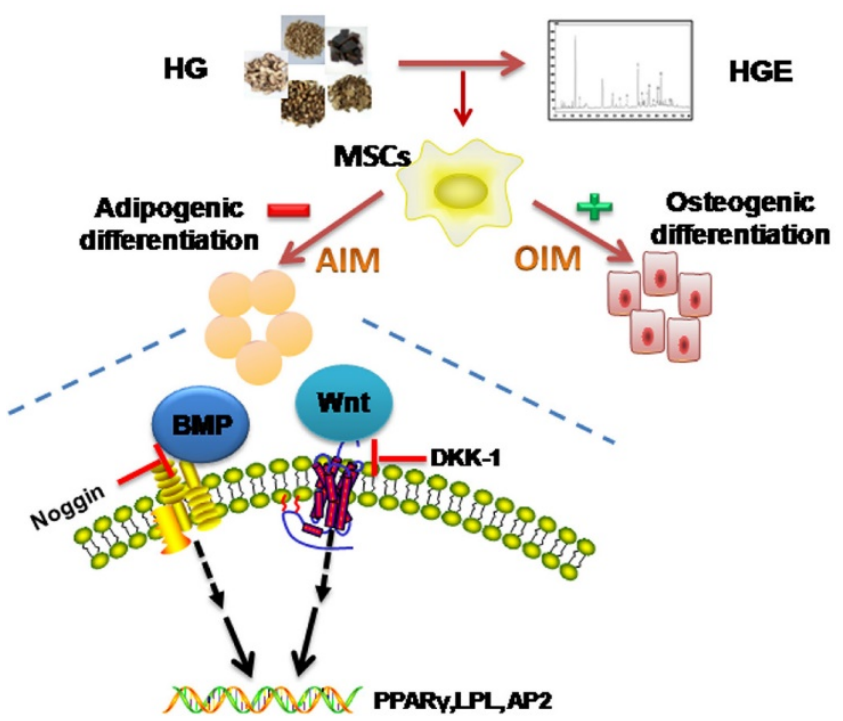

Figure 9. Schematic illustration showing putative-signaling pathways involved in the effect of HGE on the adipogenic differentiation of BMSCs. HGE activates BMP2 and Wnt pathways leading to the transcription of PPAR, LPL and AP2, ultimately induced adipogenic differentiation.

\section{Supplementary Material}

Supplementary figures.

http://www.ijbs.com/v13p0480s1.pdf

\section{Abbreviations}

AIM: Adipogenic induction medium; ALP: Alkaline phosphatase; AP2: adipocyteprotein2; BMP: Bone Morphogenetic Protein; BMSCs: bone marrow stromal cells; HGE: ethyl acetate fraction of Huogu Formula; LPL: lipoprotein lipase; OIM: Osteogenesis induction medium; ONFH: osteonecrosis of femoral head; PPAR: peroxisome proliferators-activated receptor.

\section{Acknowledgments}

This study was supported by grants from Beijing Municipal Science and Technology Commission
(No.Z151100003815028) and National Natural Science Foundation of China (No.81173417).

\section{Author Contributions}

Lin N., Chen WH. and Kong XY conceived and designed the experiments; Li XM, Wan HY, Zhu LL and Zhu J, Qin QX and Kong XY performed the in vitro experiments; Liu CF, Wang QQ and He LH conducted the in vivo experiments; Zhang $\mathrm{C}$ did the chemical analysis; $\mathrm{Wu} X$ and Liu $\mathrm{CL}$ analyzed the data; Kong $\mathrm{XY}$ wrote the manuscript and $\mathrm{N}$ Lin reviewed it.

\section{Competing Interests}

The authors have declared that no competing interest exists.

\section{References}

1. Dilisio MF. Osteonecrosis following short-term, low-dose oral corticosteroids: a population-based study of 24 million patients. Orthopedics. 2014; 37: e631-6.

2. Gebhard KL, Maibach HI. Relationship between systemic corticosteroids and osteonecrosis. American journal of clinical dermatology. 2001; 2: 377-88.

3. Miyanishi K, Yamamoto T, Irisa T, Yamashita A, Jingushi S, Noguchi Y, et al. Bone marrow fat cell enlargement and a rise in intraosseous pressure in steroid-treated rabbits with osteonecrosis. Bone. 2002; 30: 185-90.

4. Li X, Jin L, Cui Q, Wang GJ, Balian G. Steroid effects on osteogenesis through mesenchymal cell gene expression. Osteoporosis international : a journal established as result of cooperation between the European Foundation for Osteoporosis and the National Osteoporosis Foundation of the USA. 2005; 16: 101-8.

5. Wang GJ, Cui Q, Balian G. The Nicolas Andry award. The pathogenesis and prevention of steroid-induced osteonecrosis. Clinical orthopaedics and related research. 2000: 295-310.

6. Li JP, Chen S, Peng H, Zhou JL, Fang HS. Pulsed electromagnetic fields protect the balance between adipogenesis and osteogenesis on steroid-induced osteonecrosis of femoral head at the pre-collapse stage in rats. Bioelectromagnetics. 2014; 35: 170-80.

7. Kang P, Gao H, Pei F, Shen B, Yang J, Zhou Z. Effects of an anticoagulant and a lipid-lowering agent on the prevention of steroid-induced osteonecrosis in rabbits. International journal of experimental pathology. 2010; 91: 235-43.

8. Pengde K, Fuxing P, Bin S, Jing Y, Jingqiu C. Lovastatin inhibits adipogenesis and prevents osteonecrosis in steroid-treated rabbits. Joint, bone, spine : revue du rhumatisme. 2008; 75: 696-701.

9. Chen WH, Zhou Y, He HJ, Liu DB, Wang ZY, Wang RT, et al. Treating early-to-middle stage nontraumatic osteonecrosis of femoral head patients by jianpi huogu recipe: a retrospective study. Zhongguo Zhong xi yi jie he za zhi Zhongguo Zhongxiyi jiehe zazhi $=$ Chinese journal of integrated traditional and Western medicine / Zhongguo Zhong xi yi jie he xue hui, Zhongguo Zhong yi yan jiu yuan zhu ban. 2013; 33: 1054-8.

10. Chen WH, Kong XY, Wan R, Xiao CS, Li L, Wang ZY, et al. Effects of huogu I formula (I) on correlated factors of bone regeneration in chickens with steroid-induced necrosis of femoral head. Chinese journal of integrative medicine. 2012 ; 18 : 378-84.

11. Wan R, Lin SF, Lin N, Xiao CS, Kong XY, Chen WH. Effects of different Chinese drugs on bone histomorphology of hormone induced femoral head necrosis. Zhongguo gu shang $=$ China journal of orthopaedics and traumatology. 2010; 23: 915-9.

12. Jiang $\mathrm{Y}$, Liu D, Kong $\mathrm{X}, \mathrm{Xu} \mathrm{Y}$, Chen W, Lin N. Huogu I formula prevents steroid-induced osteonecrosis in rats by down-regulating PPARgamma expression and activating wnt/LRP5/ beta-catenin signaling. Journal of traditional Chinese medicine $=$ Chung $\mathrm{i}$ tsa chih ying wen pan / sponsored by All-China Association of Traditional Chinese Medicine, Academy of Traditional Chinese Medicine. 2014; 34: 342-50.

13. Taipaleenmaki H, Abdallah BM, AlDahmash A, Saamanen AM, Kassem M. Wnt signalling mediates the cross-talk between bone marrow derived pre-adipocytic and pre-osteoblastic cell populations. Experimental cell research 2011; 317: 745-56.

14. Commission NP. Pharmacopoeia of the People's Republic of China. 2 ed. Beijing: Chemical Industry Press; 2015.

15. Maniatopoulos C, Sodek J, Melcher AH. Bone formation in vitro by stromal cells obtained from bone marrow of young adult rats. Cell and tissue research. 1988; 254: 317-30.

16. Soleimani M, Nadri S. A protocol for isolation and culture of mesenchymal stem cells from mouse bone marrow. Nature protocols. 2009; 4: 102-6. 
17. Saintier D, Khanine V, Uzan B, Ea HK, de Vernejoul MC, Cohen-Solal ME. Estradiol inhibits adhesion and promotes apoptosis in murine osteoclasts in vitro. The Journal of steroid biochemistry and molecular biology. 2006; 99: 165-73.

18. Backesjo CM, Li Y, Lindgren U, Haldosen LA. Activation of Sirt1 decreases adipocyte formation during osteoblast differentiation of mesenchymal stem cells. Journal of bone and mineral research : the official journal of the American Society for Bone and Mineral Research. 2006; 21: 993-1002.

19. Lee SY, Lee JH, Kim JY, Bae YC, Suh KT, Jung JS. BMP2 increases adipogenic differentiation in the presence of dexamethasone, which is inhibited by the treatment of TNF-alpha in human adipose tissue-derived stromal cells. Cellular physiology and biochemistry : international journal of experimental cellular physiology, biochemistry, and pharmacology. 2014; 34: 1339-50.

20. Weinstein RS. Glucocorticoid-induced osteonecrosis. Endocrine. 2012; 41: 183-90.

21. Liu C, Janke LJ, Kawedia JD, Ramsey LB, Cai X, Mattano LA, Jr., et al. Asparaginase Potentiates Glucocorticoid-Induced Osteonecrosis in a Mouse Model. PloS one. 2016; 11: e0151433.

22. Sheng H, Sheng CJ, Cheng XY, Zhang G, Lee KM, Leung KS, et al. Pathomorphological changes of bone marrow adipocytes in process of steroid-associated osteonecrosis. International journal of clinical and experimental pathology. 2013; 6: 1046-50.

23. Sheng HH, Zhang GG, Cheung WH, Chan CW, Wang YX, Lee KM, et al. Elevated adipogenesis of marrow mesenchymal stem cells during early steroid-associated osteonecrosis development. Journal of orthopaedic surgery and research. 2007; $2: 15$

24. Ge C, Cawthorn WP, Li Y, Zhao G, MacDougald OA, Franceschi RT. Reciprocal Control of Osteogenic and Adipogenic Differentiation by ERK/MAP Kinase Phosphorylation of Runx2 and PPARgamma Transcription Factors. Journal of cellular physiology. 2015.

25. Beresford JN, Bennett JH, Devlin C, Leboy PS, Owen ME. Evidence for an inverse relationship between the differentiation of adipocytic and osteogenic cells in rat marrow stromal cell cultures. Journal of cell science. 1992; $102(\mathrm{Pt}$ 2): 341-51

26. James AW. Review of Signaling Pathways Governing MSC Osteogenic and Adipogenic Differentiation. Scientifica. 2013; 2013: 684736

27. Bethel M, Chitteti BR, Srour EF, Kacena MA. The changing balance between osteoblastogenesis and adipogenesis in aging and its impact on hematopoiesis. Current osteoporosis reports. 2013; 11: 99-106.

28. Naveiras O, Nardi V, Wenzel PL, Hauschka PV, Fahey F, Daley GQ. Bone-marrow adipocytes as negative regulators of the haematopoietic microenvironment. Nature. 2009; 460: 259-63.

29. Sugimura R, Li L. Shifting in balance between osteogenesis and adipogenesis substantially influences hematopoiesis. Journal of molecular cell biology. 2010; 2: 61-2.

30. Muruganandan S, Sinal CJ. The impact of bone marrow adipocytes on osteoblast and osteoclast differentiation. IUBMB life. 2014

31. Tzameli I, Fang $\mathrm{H}$, Ollero $\mathrm{M}$, Shi $\mathrm{H}$, Hamm JK, Kievit $\mathrm{P}$, et al. Regulated production of a peroxisome proliferator-activated receptor-gamma ligand during an early phase of adipocyte differentiation in 3T3-L1 adipocytes. The Journal of biological chemistry. 2004; 279: 36093-102.

32. Lazarenko OP, Rzonca SO, Suva LJ, Lecka-Czernik B. Netoglitazone is a PPAR-gamma ligand with selective effects on bone and fat. Bone. 2006; 38: 74-84.

33. Yuan Z, Li O, Luo S, Liu Z, Luo D, Zhang B, et al. PPARgamma and Wnt Signaling in Adipogenic and Osteogenic Differentiation of Mesenchymal Stem Cells. Current stem cell research \& therapy. 2015.

34. Kang Q, Song WX, Luo Q, Tang N, Luo J, Luo X, et al. A comprehensive analysis of the dual roles of BMPs in regulating adipogenic and osteogenic differentiation of mesenchymal progenitor cells. Stem cells and development. 2009; 18: 545-59.

35. Zhang X, Guo J, Zhou Y, Wu G. The roles of bone morphogenetic proteins and their signaling in the osteogenesis of adipose-derived stem cells. Tissue engineering Part B, Reviews. 2014; 20: 84-92. 